\title{
Classical model of a delayed-choice quantum eraser
}

\author{
Brian R. La Cour $\odot^{*}$ and Thomas W. Yudichak $\odot$ \\ Applied Research Laboratories, The University of Texas at Austin, P.O. Box 8029, Austin 78713-8029, Texas
}

(Received 9 January 2021; accepted 27 May 2021; published 9 June 2021)

\begin{abstract}
Wheeler's delayed-choice experiment was conceived to illustrate the paradoxical nature of wave-particle duality in quantum mechanics. In the experiment, quantum light can exhibit either wavelike interference patterns or particlelike anticorrelations, depending upon the (possibly delayed) choice of the experimenter. A variant known as the quantum eraser uses entangled light to recover the lost interference in a seemingly nonlocal and retrocausal manner. Although it is believed that this behavior is incompatible with classical physics, here we show that, using postselection, the observed quantum phenomena can be reproduced by adopting a simple deterministic detector model and supposing the existence of a random zero-point electromagnetic field.
\end{abstract}

DOI: 10.1103/PhysRevA.103.062213

\section{INTRODUCTION}

Wave-particle duality is one of the oldest and most perplexing aspects of quantum theory [1]. Although the wavelike nature of light had been well established by the 19th century, experiments of the early 20th century brought about the notion of light as a particle, what we now call a photon [2-4]. Maintaining this notion of light as being composed of discrete particles can, however, be rather paradoxical at times, as numerous real and gedanken experiments have shown [5-10].

One particular experiment that has captured recent interest and attention is the delayed-choice quantum eraser. First conceived by Scully and Drühl in 1982 [11], the quantum eraser is a variant of Wheeler's delayed choice experiment in which measurements on one of a pair of entangled light beams are used to recover an interference pattern, and hence wavelike behavior, that would otherwise be lost with the introduction of which-way path information in a Mach-Zehnder interferometer [12-16]. For Wheeler, the delayed-choice experiment was an argument for antirealism, the notion that quantum objects, such as photons, do not have definite, intrinsic properties that are independent of the measurement context [17]. Some, however, have interpreted the results of delayed-choice quantum eraser experiments as evidence for a form of retrocausality [18].

More recently, a series of delayed-choice experiments has been performed that rule out a certain class of nonretrocausal hidden-variable models described by Chaves, Lemos, and Pienaar [19]. The general model they describe can provide a causal description of the standard delayed-choice exper-

\footnotetext{
*blacour@arlut.utexas.edu

Published by the American Physical Society under the terms of the Creative Commons Attribution 4.0 International license. Further distribution of this work must maintain attribution to the author(s) and the published article's title, journal citation, and DOI.
}

iment, but it fails to describe a variant of this experiment using variable phase delays in the arms of the interferometer. This variant has been the subject of recent experimental investigations, which are consistent with theoretical predictions [20-22]. These experiments place certain dimensional restrictions on the class of nonretrocausal hidden-variable models that can be consistent with theory and observations.

In this paper, we revisit Wheeler's delayed-choice experiment, its recent experimental variants, and the more elaborate quantum eraser experiment within the context of a simple, physically motivated classical model $[23,24]$. Although loophole-free experiments have already been performed to rule out local realism [25-27], the identification of precisely which phenomena defy a classical interpretation remains an open question and one of practical relevance to ensure the security and efficacy of emergent quantum technologies. Our approach is modeled after stochastic electrodynamics (SED) in assuming a reified (i.e., nonvirtual) zero-point field (ZPF) corresponding to the vacuum state $[28,29]$. A significant departure from standard SED in our approach is the introduction of a deterministic model of detectors using an amplitude threshold crossing scheme. We find that these simple assumptions, combined with standard experimental postselection and data analysis techniques, adequately describe the observed quantum phenomena.

The structure of the paper is as follows: In Sec. II we consider a simple delayed-choice experiment using weak coherent light as a notional single-photon source. We replace the coherent light with a source of entangled light in Sec. III, within the context of a quantum eraser experiment, and demonstrate how postselection, not causality, is the mechanism whereby path information is effectively erased. With these two results established, we revisit the theoretical arguments of Chaves and Bowles in Sec. V and argue that their assumptions are overly restrictive. Section VI considers a variant of these experiments using entangled light sources and shows that these, too, can be understood within a classical framework. Finally, we summarize our conclusions in Sec. VII. Figures and numerical experiments were created 
and performed using a custom simulation tool, the Virtual Quantum Optics Laboratory (VQOL) [30].

\section{SIMPLE DELAYED-CHOICE EXPERIMENT}

Consider the Mach-Zehnder interferometer of Fig. 1. A laser (LAS) provides a source of coherent, horizontally polarized light that is strongly attenuated by a neutral density filter (NDF) before entering the first beam splitter (BS1). Under our model, the laser light exiting the NDF is represented by a stochastic Jones vector of the form

$$
\boldsymbol{a}=\left(\begin{array}{l}
a_{H} \\
a_{V}
\end{array}\right)=\left(\begin{array}{l}
\alpha \\
0
\end{array}\right)+\sigma_{0}\left(\begin{array}{c}
z_{1 H} \\
z_{1 V}
\end{array}\right),
$$

where $\alpha \in \mathbb{C}$ describes the mean amplitude and phase of the light, $\sigma_{0}=1 / \sqrt{2}$ is the scale of the ZPF (corresponding to a modal energy of $\frac{1}{2} \hbar \omega$ ), and $z_{1 H}$ and $z_{1 V}$ are independent standard complex Gaussian random variables. (We say that $z$ is a standard complex Gaussian random variable if $E[z]=0$, $\mathrm{E}\left[|z|^{2}\right]=1$, and $\mathrm{E}\left[z^{2}\right]=0$ ). Note that $z_{1 H}$ and $z_{1 V}$ play the role of hidden variables. This model is mathematically equivalent to the corresponding quantum coherent state $|\alpha\rangle \otimes|0\rangle$ whose Wigner function is a bivariate Gaussian probability density function identical to that of $\boldsymbol{a}$. The fact that we are using explicit random variables in our representation amounts to a reification of the ZPF. The effect of the NDF is to ensure that $|\alpha| \ll 1$.

Passage through the first 50/50 beam splitter (denoted by BS1) splits the beam into two orthogonal spatial modes, a right-traveling mode (denoted by $\rightarrow$ ) and a down-traveling mode (denoted by $\downarrow$ ). In addition, there is a down-traveling vacuum mode that enters the top input port of $\mathrm{BS} 1$, represented by the independent stochastic Jones vector

$$
\boldsymbol{b}=\left(\begin{array}{l}
b_{H} \\
b_{V}
\end{array}\right)=\sigma_{0}\left(\begin{array}{l}
z_{2 H} \\
z_{2 V}
\end{array}\right)
$$

where $z_{2 H}$ and $z_{2 V}$ are independent standard complex Gaussian random variables (which are also independent of $z_{1 H}$ and $z_{1 V}$ ). The two spatial modes may be represented by a pair of

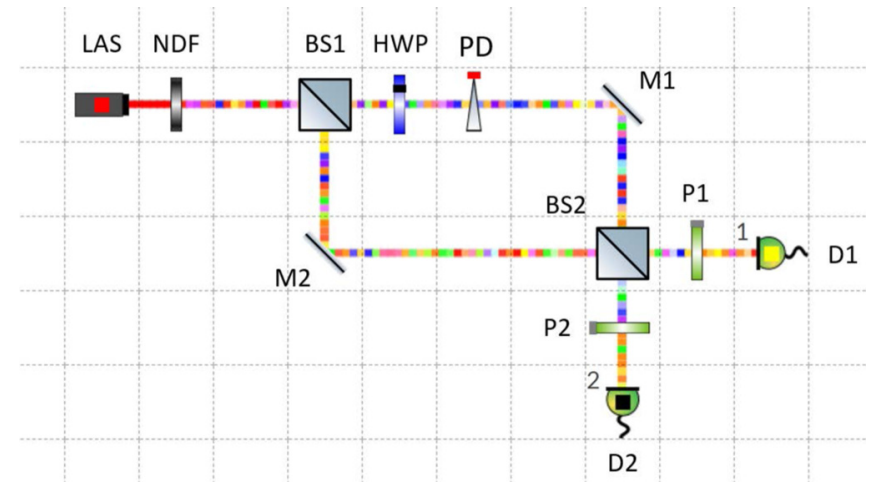

FIG. 1. VQOL experimental setup for a simple delayed-choice experiment. The different colors (or shades of gray) in the beams correspond to different polarizations. stacked Jones vectors as follows:

$$
\left[\begin{array}{c}
a_{H} \\
a_{V} \\
-- \\
b_{H} \\
b_{V}
\end{array}\right] \stackrel{\text { BS1 }}{\longrightarrow} \frac{1}{\sqrt{2}}\left[\begin{array}{c}
a_{H}+b_{H} \\
a_{V}+b_{V} \\
---- \\
a_{H}-b_{H} \\
a_{V}-b_{V}
\end{array}\right] .
$$

Note that the second term on the right-hand side is again a standard complex Gaussian random vector, owing to the unitarity of the beam splitter transformation.

The right-traveling beam next undergoes a transformation via a half-wave plate (denoted by HWP) with a fast-axis angle $\theta \in[0, \pi / 4]$ relative to the horizontal axis. It subsequently undergoes a phase delay (denoted by PD) that applies a global phase angle $\phi \in[0,2 \pi]$. Finally, a pair of mirrors (denoted by M1 and M2) swap the two spatial modes. The resulting stacked Jones vector after these three transformations is now

$$
\frac{1}{\sqrt{2}}\left[\begin{array}{c}
a_{H}+b_{H} \\
a_{V}+b_{V} \\
---- \\
a_{H}-b_{H} \\
a_{V}-b_{V}
\end{array}\right] \stackrel{\text { HWP, PD, M1, M2 }}{\longrightarrow}\left[\begin{array}{c}
a_{H}^{\prime} \\
a_{V}^{\prime} \\
-- \\
b_{H}^{\prime} \\
b_{V}^{\prime}
\end{array}\right],
$$

where $a_{H}^{\prime}=\left(a_{H}-b_{H}\right) / \sqrt{2}, a_{V}^{\prime}=\left(a_{V}-b_{V}\right) / \sqrt{2}$, and

$$
\begin{aligned}
& b_{H}^{\prime}=\frac{e^{i \phi}}{\sqrt{2}}\left[\cos 2 \theta\left(a_{H}+b_{H}\right)+\sin 2 \theta\left(a_{V}+b_{V}\right)\right], \\
& b_{V}^{\prime}=\frac{e^{i \phi}}{\sqrt{2}}\left[\sin 2 \theta\left(a_{H}+b_{H}\right)-\cos 2 \theta\left(a_{V}+b_{V}\right)\right] .
\end{aligned}
$$

In the absence of the second beam splitter (denoted by BS2), the Jones vectors $\left(a_{H}^{\prime}, a_{V}^{\prime}\right)^{\top}$ and $\left(b_{H}^{\prime}, b_{V}^{\prime}\right)^{\top}$ will determine if a detection is made on the right-traveling mode (by detector D1) or the down-traveling mode (by detector D2). We adopt an amplitude threshold crossing scheme as a model to determine whether a given detector makes a detection (or "clicks") [23]. Under this scheme, a detector clicks if the amplitude of either the horizontal or the vertical polarization component of the impinging beam falls above a given threshold, $\gamma \geqslant 0$.

Each detector has placed before it a polarizer (denoted by P1 and P2) oriented to admit horizontally polarized light. A polarizer can be modeled as a polarizing beam splitter for which one of the output ports is ignored [31]. Consequently, an independent vacuum mode will be present in the second input port, resulting in the transformation

$$
\left(\begin{array}{c}
a_{H}^{\prime} \\
a_{V}^{\prime}
\end{array}\right) \stackrel{\mathrm{P} 1}{\longrightarrow}\left(\begin{array}{c}
a_{H}^{\prime} \\
\sigma_{0} z_{1 V}^{\prime}
\end{array}\right),
$$

where $z_{1 V}^{\prime}$ is an independent standard complex Gaussian random variable corresponding to the vacuum mode of the notional second input port. Similarly, passage through P2 will result in the transformation

$$
\left(\begin{array}{c}
b_{H}^{\prime} \\
b_{V}^{\prime}
\end{array}\right) \stackrel{\mathrm{P} 2}{\longrightarrow}\left(\begin{array}{c}
b_{H}^{\prime} \\
\sigma_{0} z_{2 V}^{\prime}
\end{array}\right),
$$

where $z_{2 V}^{\prime}$ is, again, an independent standard complex Gaussian random variable.

Thus, detector D1 clicks, according to this model, if the random variables $z_{1 H}, z_{1 V}, z_{2 H}, z_{2 V}, z_{1 V}^{\prime}$, and $z_{2 V}^{\prime}$ are such that 
they fall with the event set

$$
D_{1}=\left\{\left|a_{H}^{\prime}\right|>\gamma \text { or }\left|\sigma_{0} z_{1 V}^{\prime}\right|>\gamma\right\} .
$$

The deterministic nature of the detector model lies in the fact that a click occurs only when a particular random realization of the hidden variables is such that at least one of the two amplitudes falls above the threshold $\gamma$. Similarly, detector D2 clicks under the event set

$$
D_{2}=\left\{\left|b_{H}^{\prime}\right|>\gamma \text { or }\left|\sigma_{0} z_{2 V}^{\prime}\right|>\gamma\right\}
$$

Clearly, the probabilities for these events are independent of the phase angle $\phi$, since only the amplitudes of $a_{H}^{\prime}$ and $b_{H}^{\prime}$ are considered. Thus, no interference effects would be observed by simply varying $\phi$.

One may consider the alternative counterfactual case in which the second beam splitter, BS2, is present. In this case, we have a further transformation,

$$
\left[\begin{array}{c}
a_{H}^{\prime} \\
a_{V}^{\prime} \\
-- \\
b_{H}^{\prime} \\
b_{V}^{\prime}
\end{array}\right] \stackrel{\mathrm{BS} 2}{\longrightarrow} \frac{1}{\sqrt{2}}\left[\begin{array}{c}
a_{H}^{\prime}+b_{H}^{\prime} \\
a_{V}^{\prime}+b_{V}^{\prime} \\
---- \\
a_{H}^{\prime}-b_{H}^{\prime} \\
a_{V}^{\prime}-b_{V}^{\prime}
\end{array}\right]=\left[\begin{array}{c}
a_{H}^{\prime \prime} \\
a_{V}^{\prime \prime} \\
-- \\
b_{H}^{\prime \prime} \\
b_{V}^{\prime \prime}
\end{array}\right] .
$$

Detector D1 now clicks under the event

$$
D_{1}^{\prime}=\left\{\left|a_{H}^{\prime \prime}\right|>\gamma \text { or }\left|\sigma_{0} z_{1 V}^{\prime}\right|>\gamma\right\},
$$

while detector D2 clicks under the event

$$
D_{2}^{\prime}=\left\{\left|b_{H}^{\prime \prime}\right|>\gamma \text { or }\left|\sigma_{0} z_{2 V}^{\prime}\right|>\gamma\right\}
$$

Both $a_{H}^{\prime \prime}$ and $b_{H}^{\prime \prime}$ contain a relative phase term, resulting in a dependence on $\phi$ for the probabilities of the two events. Thus, the addition or removal of the second beam splitter may causally create or destroy, respectively, an interference pattern, even if this action is taken well after the light has passed through the first beam splitter.

We note this general qualitative behavior is itself unremarkable and may be observed more directly in the intensities of classical light (for which $|\alpha| \gg 1$ or, equivalently, $\sigma_{0} \approx 0$ ), as in this regime we find

$$
\begin{gathered}
\left|a_{H}^{\prime}\right|^{2} \approx \frac{1}{2}|\alpha|^{2}, \\
\left|a_{H}^{\prime \prime}\right|^{2} \approx \frac{1}{4}|\alpha|^{2}\left|1+e^{i \phi} \cos 2 \theta\right|^{2} .
\end{gathered}
$$

We further note that nonzero values of $\theta$ can provide whichway information, insofar as they may completely destroy, for $\theta=\pi / 2$, or merely reduce, for $0<\theta<\pi / 2$, the intensity inference pattern. To observe the more subtle particlelike behavior, we must consider the statistical properties of detection events, which we shall now do.

The random variables $a_{H}^{\prime}$ and $b_{H}^{\prime}$ obey a proper complex Gaussian distribution with expectation values of

$$
\begin{gathered}
\mathrm{E}\left[a_{H}^{\prime}\right]=\frac{\alpha}{\sqrt{2}}, \\
\mathrm{E}\left[b_{H}^{\prime}\right]=\frac{\alpha}{\sqrt{2}} \cos 2 \theta
\end{gathered}
$$

and a common variance of $\sigma_{0}^{2}$. They are furthermore independent, owing to the unitarity of the transformations involved. Thus, $\left|a_{H}^{\prime}\right|$ and $\left|b_{H}^{\prime}\right|$ each follow a Rician distribution [32]. Since, furthermore, $z_{1 V}$ and $z_{2 V}$ are also independent, the

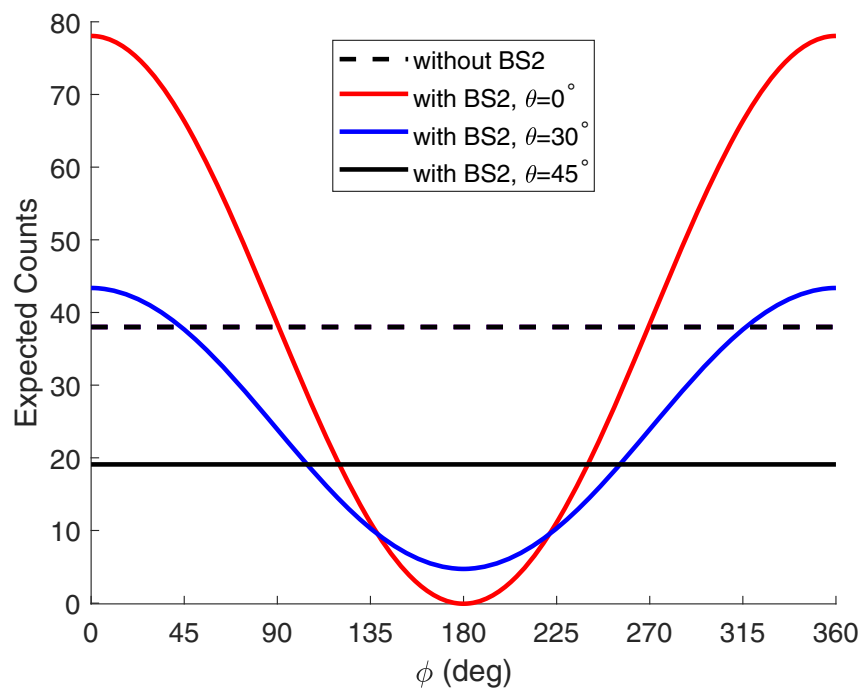

FIG. 2. Plot of the expected counts, minus dark counts, for a delayed-choice experiment versus the phase delay angle $\phi$ in the upper arm of the interferometer. The dashed line corresponds to when BS2 is removed, and the solid lines correspond to when BS2 is present. Interference patterns are observed when there is no which-way information $\left[\theta=0^{\circ}\right.$, red (light gray) line] or only partial which-way information $\left[\theta=30^{\circ}\right.$, blue (dark gray) line]. The interference pattern vanishes with complete which-way information $\left(\theta=45^{\circ}\right.$, black solid line $)$.

probability of a single click on detector D1 (and not D2) is given by

$$
p_{1}(\theta, \phi)=\operatorname{Pr}\left[D_{1} \cap \bar{D}_{2}\right]=\operatorname{Pr}\left[D_{1}\right]\left(1-\operatorname{Pr}\left[D_{2}\right]\right),
$$

where

$$
\begin{gathered}
\operatorname{Pr}\left[D_{1}\right]=1-F\left(\frac{\alpha}{\sqrt{2}}\right) F(0), \\
\operatorname{Pr}\left[D_{2}\right]=1-F\left(\frac{\alpha}{\sqrt{2}} \cos 2 \theta\right) F(0),
\end{gathered}
$$

and $F(\alpha)=\operatorname{Pr}\left[\left|\alpha+z_{H}\right| \leqslant \gamma\right]$ is given by the Marcum $Q$ function as $[23,33]$

$$
F(\alpha)=1-Q_{1}\left(\sqrt{2}|\alpha| / \sigma_{0}, \sqrt{2} \gamma / \sigma_{0}\right) .
$$

Note that $Q_{1}\left(0, \sqrt{2} \gamma / \sigma_{0}\right)=e^{-\gamma^{2} / \sigma_{0}^{2}}$.

A similar analysis may be used when the second beam splitter is in place, albeit using $a_{H}^{\prime \prime}$ and $b_{H}^{\prime \prime}$ instead. The probability of a single click on detector D1 is now

$$
p_{1}^{\prime}(\theta, \phi)=\operatorname{Pr}\left[D_{1}^{\prime}\right]\left(1-\operatorname{Pr}\left[D_{2}^{\prime}\right]\right),
$$

where

$$
\begin{aligned}
& \operatorname{Pr}\left[D_{1}^{\prime}\right]=1-F\left(\frac{\alpha}{2}\left(1+e^{i \phi} \cos 2 \theta\right)\right) F(0), \\
& \operatorname{Pr}\left[D_{2}^{\prime}\right]=1-F\left(\frac{\alpha}{2}\left(1-e^{i \phi} \cos 2 \theta\right)\right) F(0) .
\end{aligned}
$$

In Fig. 2 we have plotted the expected number of counts, in excess of the dark counts, for the cases with and without BS2 present and for different values of $\theta$. The number of notional trials was taken to be $N=10^{6}$. The laser and NDF were taken to be such that $\alpha=0.1$, and the detectors were 
such that $\gamma=1.95$, corresponding to a dark count probability of $p_{d}=0.001$. Without BS2 present, the expected counts are $N p_{1}(\theta, \phi)-N p_{d}$; with $\mathrm{BS} 2$ in place, they are $N p_{1}^{\prime}(\theta, \phi)-$ $N p_{d}$. The HWP fast-axis angle, $\theta$, was taken to be either $0^{\circ}$, $30^{\circ}$, or $45^{\circ}$, corresponding to no, partial, or complete whichway information, respectively.

As expected, there is no interference pattern when BS2 is removed. With $\mathrm{BS} 2$ in place, there is a strong interference pattern when there is no which-way information (i.e., $\theta=0^{\circ}$ ). When partial which-way information is available, corresponding to a nonzero vertical component of the mean polarization, the interference pattern is diminished but remains discernible. If complete which-way information is available, corresponding to a mean vertical polarization in the upper arm of the interferometer, the interference pattern is diminished to the point of being no longer present. These effects are completely causal and arise from the interference of classical, albeit stochastic, waves. The particlelike behavior, manifested by single-detection events, is a consequence of the low intensity of the input beam, for which detections on both detectors are rare, and the fact that we have removed nondetection events through postselection.

In the parameter regime we have chosen, the interference pattern matches a scaled and shifted version of the $\cos ^{2}(\phi / 2)$ probability predicted by quantum mechanics for a singlephoton state. Larger values of $\alpha$ may exhibit deviations from this prediction, as the corresponding coherent state $|\alpha\rangle$ may no longer be considered a good approximation of a vacuumplus-single-photon state.

As a final note, the ability to causally model a standard delayed-choice experiment has already been demonstrated by Chaves et al. [19]. In Secs. V and VI we consider a more sophisticated version of the delayed-choice experiment, as suggested by Ref. [19], and demonstrate how it, too, can be explained by our classical model.

\section{DELAYED-CHOICE QUANTUM ERASER}

Wheeler's delayed-choice experiment can be changed to a quantum eraser experiment by replacing the laser and the NDF with an entanglement source (ENT). The experiment is illustrated in Fig. 3, where we have also added a third polarizer and a third detector, denoted P3 and D3, respectively. Unlike the other two polarizers, $\mathrm{P} 3$ is rotated by $45^{\circ}$ so as to admit diagonally polarized light.

The entanglement source is modeled classically as a typeI parametric down-conversion process for which the inputs states are a pump laser (not shown) and classically modeled vacuum modes from the ZPF [24]. The Jones vectors for the right-traveling $(\rightarrow)$ and left-traveling $(\leftarrow)$ spatial modes of the entanglement source, denoted $\boldsymbol{a}$ and $\boldsymbol{c}$, respectively, are given by

$$
\boldsymbol{a}=\left(\begin{array}{l}
a_{H} \\
a_{V}
\end{array}\right)=\sigma_{0}\left(\begin{array}{l}
z_{1 H} \cosh r+z_{3 H}^{*} \sinh r \\
z_{1 V} \cosh r+z_{3 V}^{*} \sinh r
\end{array}\right)
$$

and

$$
\boldsymbol{c}=\left(\begin{array}{l}
c_{H} \\
c_{V}
\end{array}\right)=\sigma_{0}\left(\begin{array}{l}
z_{3 H} \cosh r+z_{1 H}^{*} \sinh r \\
z_{3 V} \cosh r+z_{1 V}^{*} \sinh r
\end{array}\right),
$$

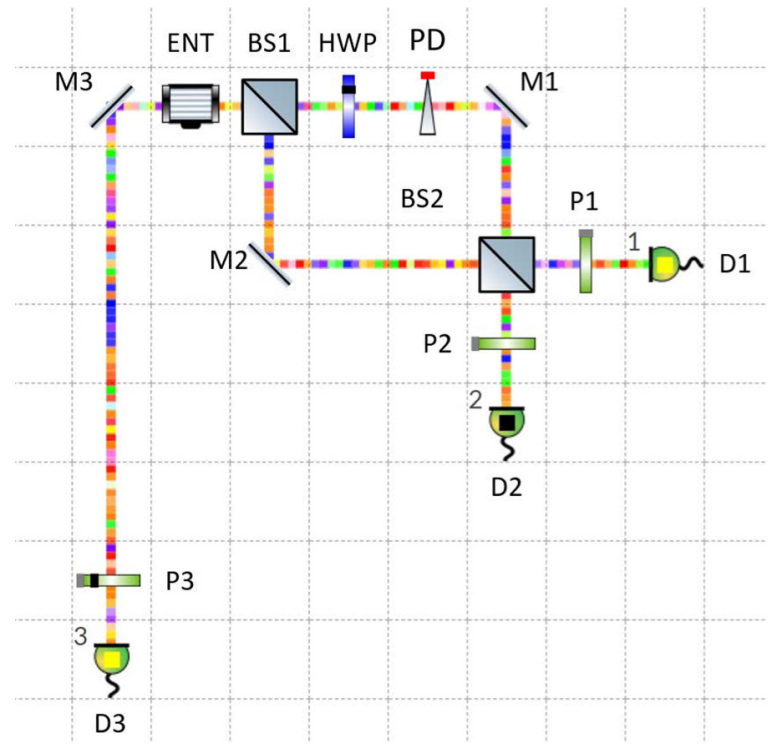

FIG. 3. VQOL experimental setup for a delayed-choice quantum eraser experiment. The entanglement source is labeled ENT.

where $z_{1 H}, z_{1 V}, z_{3 H}$ and $z_{3 V}$ are independent standard complex Gaussian random variables and $r \geqslant 0$ is the squeezing strength of the entanglement source. We note that $\boldsymbol{a}$ and $\boldsymbol{c}$ are statistically dependent for $r>0$.

The joint probability density function of $\boldsymbol{a}$ and $\boldsymbol{c}$ is identical to the Gaussian Wigner function of a four-mode entangled squeezed state [34]. If $r \ll 1$, this squeezed state may be approximated as a superposition of a vacuum state and the entangled Bell state

$$
|\Psi\rangle=\frac{|H\rangle \otimes|H\rangle+|V\rangle \otimes|V\rangle}{\sqrt{2}},
$$

where the left and right kets in each tensor product indicate the spatial modes traveling to the left and right, respectively.

As before, $\boldsymbol{b}$ denotes the Jones vector of the down-traveling $(\downarrow)$ vacuum mode entering BS1 and given by

$$
\boldsymbol{b}=\left(\begin{array}{l}
b_{H} \\
b_{V}
\end{array}\right)=\sigma_{0}\left(\begin{array}{l}
z_{2 H} \\
z_{2 V}
\end{array}\right),
$$

where, again, $z_{2 H}$ and $z_{2 V}$ are independent standard complex Gaussian random variables.

The transformations of $\boldsymbol{a}$ and $\boldsymbol{b}$ through the interferometer are identical to those in the simple delayed-choice experiment discussed previously, as given by Eqs. (5) and (10). We shall again denote these by $\boldsymbol{a}^{\prime \prime}$ and $\boldsymbol{b}^{\prime \prime}$ and by $\boldsymbol{a}^{\prime}$ and $\boldsymbol{b}^{\prime}$ for the cases with and without BS2, respectively. Passage through the polarizers $\mathrm{P} 1$ and $\mathrm{P} 2$ results in the transformations

$$
\left(\begin{array}{c}
a_{H}^{\prime} \\
a_{V}^{\prime}
\end{array}\right) \stackrel{\mathrm{P} 1}{\longrightarrow}\left(\begin{array}{c}
a_{H}^{\prime} \\
\sigma_{0} z_{1 V}^{\prime}
\end{array}\right)
$$

and

$$
\left(\begin{array}{c}
b_{H}^{\prime} \\
b_{V}^{\prime}
\end{array}\right) \stackrel{\mathrm{P} 2}{\longrightarrow}\left(\begin{array}{c}
b_{H}^{\prime} \\
\sigma_{0} z_{2 V}^{\prime}
\end{array}\right),
$$

with similar transformations for $\boldsymbol{a}^{\prime \prime}$ and $\boldsymbol{b}^{\prime \prime}$. 
Upon passing through polarizer $\mathrm{P} 3, \boldsymbol{c}$ becomes

$$
\left(\begin{array}{l}
c_{H} \\
c_{V}
\end{array}\right) \stackrel{\mathrm{P} 3}{\longrightarrow} \frac{c_{D}}{\sqrt{2}}\left(\begin{array}{l}
1 \\
1
\end{array}\right)+\frac{\sigma_{0} z_{3 A}}{\sqrt{2}}\left(\begin{array}{c}
1 \\
-1
\end{array}\right),
$$

where $c_{D}=\left(c_{H}+c_{V}\right) / \sqrt{2}$ is the diagonal component of $c$ and $z_{3 A}$ is an independent standard complex Gaussian random variable corresponding to the missing antidiagonal component of the ZPF.

Individual detection events for D1, D2, and D3 for the case in which BS2 is not present may now be defined as follows:

$$
\begin{gathered}
D_{1}=\left\{\left|a_{H}^{\prime}\right|>\gamma \text { or }\left|\sigma_{0} z_{1 V}^{\prime}\right|>\gamma\right\}, \\
D_{2}=\left\{\left|b_{H}^{\prime}\right|>\gamma \text { or }\left|\sigma_{0} z_{2 V}^{\prime}\right|>\gamma\right\}, \\
D_{3}=\left\{\left|\frac{c_{D}+\sigma_{0} z_{3 A}}{\sqrt{2}}\right|>\gamma \text { or }\left|\frac{c_{D}-\sigma_{0} z_{3 A}}{\sqrt{2}}\right|>\gamma\right\} .
\end{gathered}
$$

The events $D_{1}^{\prime}, D_{2}^{\prime}$, and $D_{3}^{\prime}$, for the case in which BS2 is in place, are defined similarly by replacing $a_{H}^{\prime}$ and $b_{H}^{\prime}$ with $a_{H}^{\prime \prime}$ and $b_{H}^{\prime \prime}$. (Both $c_{D}$ are $z_{3 A}$ are, of course, unchanged).

Let us consider detection events with BS2 in place and with the HWP rotated to $\theta=45^{\circ}$, corresponding to complete which-way information. Ignoring the detections on detector D3, the probability of a single-detection on detector D1 is, as before,

$$
p_{1}^{\prime}(\phi)=\operatorname{Pr}\left[D_{1}^{\prime} \cap \bar{D}_{2}^{\prime}\right] .
$$

If we postselect on events for which detector D3 also clicks, this probability becomes

$$
p_{13}^{\prime}(\phi)=\operatorname{Pr}\left[D_{1}^{\prime} \cap \bar{D}_{2}^{\prime} \mid D_{3}^{\prime}\right] .
$$

We do not have a closed-form expression for the joint distribution of $\boldsymbol{a}$ and $\boldsymbol{c}$; however, these probabilities may be estimated numerically. Taking $\gamma=1.95$, as before, and $r=1$, we estimated the probabilities $p_{1}^{\prime}(\phi)$ and $p_{13}^{\prime}(\phi)$ for an ensemble of $N=10^{6}$ realizations. (Each realization assigns a particular set of values for the hidden variables; in VQOL, each time step generates an independent realization). The results are shown in Fig. 4. As expected, the interference pattern is recovered when we postselect on D3 detections.

In the language of quantum mechanics, we have "erased" the which-way information by collapsing the state with a projective measurement at D3. In truth, all we have done is sample from a subensemble of ZPF realizations for which there is a detection on D3. Classically, there is no causal mechanism by which detection events at D3 affect those at D1 or D2. It is merely a reflection of the pre-existing correlations between $\boldsymbol{a}$ and $\boldsymbol{c}$ and their modification as a result of postselection. Of course, it does not matter whether BS2 was inserted before or after the light had passed through BS1, so there is no need for retrocausal explanations either.

\section{QUANTUM-CONTROLLED EXPERIMENTS}

In the quantum eraser experiment of the previous section, the second beam splitter was either present or absent, resulting in the presence or absence of an interference pattern, respectively. A similar experiment was performed by Jacques et al. using a quantum random-number generator

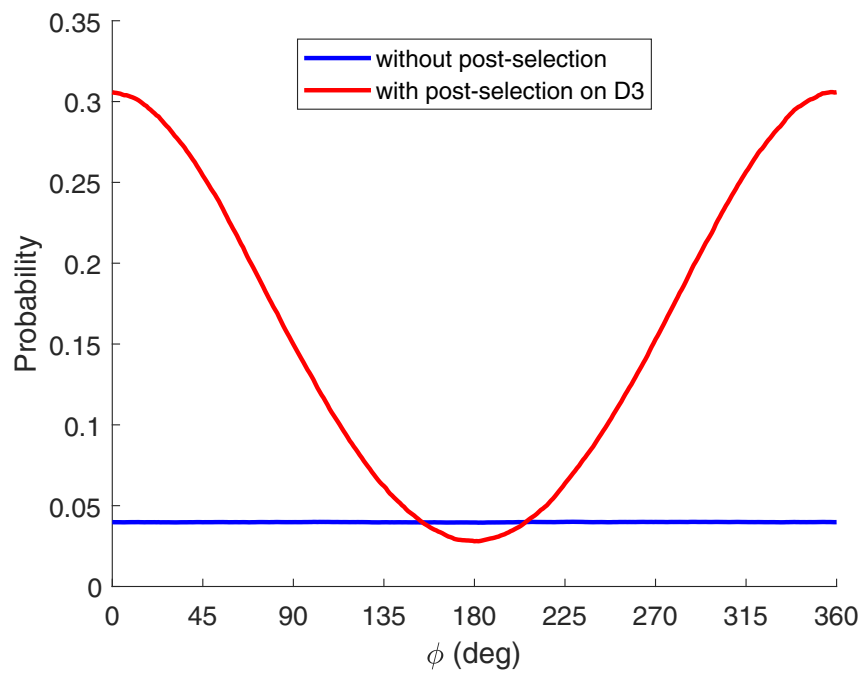

FIG. 4. Plot of the probability of a single-detection on D1 with either no conditioning on D3 [ $p_{1}^{\prime}(\phi)$, blue (dark gray) line] or with postselection of a detection on D3 [ $p_{13}^{\prime}(\phi)$, red (light gray) line].

and a classical switch [5]. Ionicioiu and Terno have suggested using a controlled quantum gate in place of a classical switch and, furthermore, have argued that this scheme may be used to rule out a certain class of hidden-variable models $[6,10]$. Subsequently, this proposal was implemented experimentally using a programmable nuclear magnetic resonance device [7], a reconfigurable integrated photonic device [8], and a polarization-dependent beam splitter (PDBS) [9]. All experiments showed the expected continuum of wavelike and particlelike behavior for different experimental settings controlling which-way path information. In this section, we consider a classical model for the PDBS experiment.

To this end we have implemented the experimental setup described in Ref. [9] with a PDBS that behaves as a 50/50 beam splitter for horizontally polarized light and is transparent (except for a swapping of spatial modes) for vertically polarized light (see Fig. 5). For Jones vectors $\boldsymbol{a}$ and $\boldsymbol{b}$ corresponding to light entering from the top and left, respectively, of the PDBS, the transformed polarization states are given by

$$
\left[\begin{array}{l}
a_{H}^{\prime} \\
a_{V}^{\prime} \\
b_{H}^{\prime} \\
b_{V}^{\prime}
\end{array}\right]=\left(\begin{array}{cccc}
\frac{1}{\sqrt{2}} & 0 & \frac{1}{\sqrt{2}} & 0 \\
0 & 0 & 0 & 1 \\
\frac{1}{\sqrt{2}} & 0 & -\frac{1}{\sqrt{2}} & 0 \\
1 & 0 & 0 & 0
\end{array}\right)\left[\begin{array}{l}
a_{H} \\
a_{V} \\
b_{H} \\
b_{V}
\end{array}\right] .
$$

As before, the phase delay (PD) is set to an angle of $\phi \in[0,2 \pi]$, and we have added a half-wave plate, HWP3, with a fast-axis angle of $\theta \in[0, \pi / 4]$. The other two halfwave plates, HWP1 and HWP2, have fast-axis angles of $\pi / 8$ in order to measure the output of the interferometer in the diagonal-antidiagonal basis.

In quantum mechanical terms, the initial entangled state is given by Eq. (26), and the final quantum state, prior to polarizing beam splitters PBS1, PBS2, and PBS3, is given by

$$
\begin{aligned}
\left|\Psi^{\prime}\right\rangle= & \frac{1}{\sqrt{2}}\left[\hat{A}_{w}^{\dagger} \cos 2 \theta+\hat{A}_{p}^{\dagger} \sin 2 \theta\right] \hat{c}_{H}^{\dagger}|\mathbf{0}\rangle \\
& +\frac{1}{\sqrt{2}}\left[\hat{A}_{w}^{\dagger} \sin 2 \theta-\hat{A}_{p}^{\dagger} \cos 2 \theta\right] \hat{c}_{V}^{\dagger}|\mathbf{0}\rangle,
\end{aligned}
$$




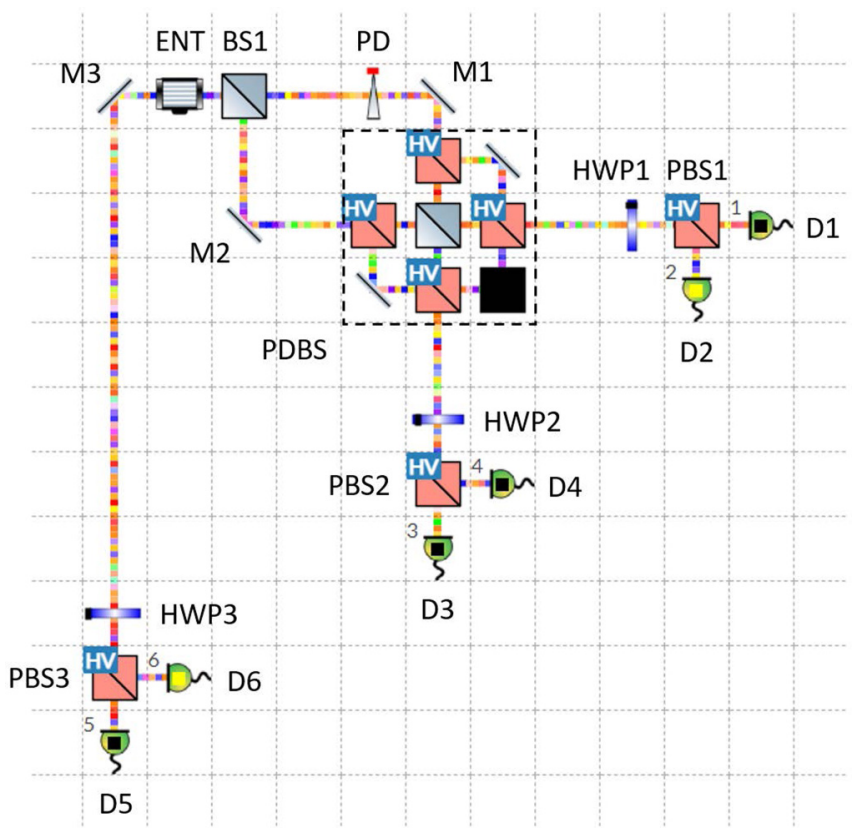

FIG. 5. VQOL experimental setup for a quantum-controlled delayed-choice quantum eraser experiment using a polarizationdependent beam splitter (PDBS). The PDBS is composed of a beam splitter surrounded by four polarizing beam splitters that transmit (reflect) horizontal (vertical) light, and a beam blocker (black square). The beam blocker absorbs ZPF modes entering from the unused input ports of the top and left PBS of the PDBS.

where $|\mathbf{0}\rangle$ is the vacuum state, $\hat{A}_{w}^{\dagger}$ is the "wave" creation operator

$$
\hat{A}_{w}^{\dagger}=\frac{1}{2 \sqrt{2}}\left[\left(1+e^{i \phi}\right)\left(\hat{a}_{H}^{\dagger}+\hat{a}_{V}^{\dagger}\right)+\left(1-e^{i \phi}\right)\left(\hat{b}_{H}^{\dagger}+\hat{b}_{V}^{\dagger}\right)\right],
$$

$\hat{A}_{p}^{\dagger}$ is the "particle" creation operator

$$
\hat{A}_{p}^{\dagger}=\frac{1}{2}\left[e^{i \phi} \hat{a}_{H}^{\dagger}-e^{i \phi} \hat{a}_{V}^{\dagger}+\hat{b}_{H}^{\dagger}-\hat{b}_{V}^{\dagger}\right],
$$

and the operators $\hat{a}_{H}^{\dagger}, \hat{a}_{V}^{\dagger}, \hat{b}_{H}^{\dagger}, \hat{b}_{V}^{\dagger}, \hat{c}_{H}^{\dagger}$, and $\hat{c}_{V}^{\dagger}$ are the creation operators for the modes corresponding to detectors D1, D2, D3, D4, D5, and D6, respectively.

In the quantum mechanical description, a single detection on either D5 or D6 results in a collapse of the wave function onto one of two subspaces, each of which is a superposition of wavelike and particlelike states. A single detection on D1 or D2, say, conditioned on a single detection on D6 would therefore have a probability of

$$
q(\theta, \phi)=\cos ^{2}(\phi / 2) \sin ^{2}(2 \theta)+\frac{1}{2} \cos ^{2}(2 \theta) .
$$

Thus, for $\theta=0(\theta=\pi / 4)$ we expect fully particlelike (wavelike) behavior. This result is equivalent to that found in Ref. [9], which uses $\theta$ and $\alpha$ in place of $\phi$ and $2 \theta$ and conditions on the horizontal, rather than vertical, mode at PBS3 due to opposite PDBS conventions.

For our classical model we take our initial states to be the Jones vectors $\boldsymbol{a}, \boldsymbol{b}$, and $\boldsymbol{c}$ as defined in Eqs. (24), (27), and (25), respectively. The final Jones vectors for the light entering
PBS1, PBS2, and PBS3 are then found to be $\boldsymbol{a}^{\prime}, \boldsymbol{b}^{\prime}$, and $\boldsymbol{c}^{\prime}$, respectively, where $\boldsymbol{a}^{\prime}$ is given by

$$
\begin{aligned}
& a_{H}^{\prime}=\frac{1+e^{i \phi}}{2 \sqrt{2}} a_{H}+\frac{e^{i \phi}}{2} a_{V}-\frac{1-e^{i \phi}}{2 \sqrt{2}} b_{H}+\frac{e^{i \phi}}{2} b_{V}, \\
& a_{V}^{\prime}=\frac{1+e^{i \phi}}{2 \sqrt{2}} a_{H}-\frac{e^{i \phi}}{2} a_{V}-\frac{1-e^{i \phi}}{2 \sqrt{2}} b_{H}-\frac{e^{i \phi}}{2} b_{V},
\end{aligned}
$$

$\boldsymbol{b}^{\prime}$ is given by

$$
\begin{aligned}
& b_{H}^{\prime}=\frac{1-e^{i \phi}}{2 \sqrt{2}} a_{H}+\frac{1}{2} a_{V}-\frac{1+e^{i \phi}}{2 \sqrt{2}} b_{H}-\frac{1}{2} b_{V}, \\
& b_{V}^{\prime}=\frac{1-e^{i \phi}}{2 \sqrt{2}} a_{H}-\frac{1}{2} a_{V}-\frac{1+e^{i \phi}}{2 \sqrt{2}} b_{H}+\frac{1}{2} b_{V},
\end{aligned}
$$

and $\boldsymbol{c}^{\prime}$ is given by

$$
\begin{aligned}
c_{H}^{\prime} & =c_{H} \cos 2 \theta+c_{V} \sin 2 \theta, \\
c_{V}^{\prime} & =c_{H} \sin 2 \theta-c_{V} \cos 2 \theta .
\end{aligned}
$$

Unlike the quantum description, there is no clear distinction between modal subspaces. For $\theta=0$, say, a single detection on D6 will occur when $\left|c_{V}\right|$ is large and $\left|c_{H}\right|$ is small. Since $\boldsymbol{c}$ and $\boldsymbol{a}$ are statistically correlated, this will tend to occur when $\left|a_{V}\right|$ is large and $\left|a_{H}\right|$ is small as well. This, in turn, implies that $a_{H}^{\prime}$ and $a_{V}^{\prime}$ are dominated by the particlelike $a_{V}$ term, versus the wavelike $a_{H}$ term, thereby giving rise to more particlelike behavior. However, the effects of the wavelike $a_{H}$ term will not be completely absent, particularly since, in amplitude, it may be up to a factor of $\sqrt{2}$ larger than the particlelike $a_{V}$ term. Thus, some wavelike interference will persist, and a mixture of wavelike and particlelike behavior will always be exhibited.

As before, we may define the individual detection events for each detector as follows:

$$
\begin{gathered}
D_{1}=\left\{\left|a_{H}^{\prime}\right|>\gamma \text { or }\left|\sigma_{0} z_{1 V}^{\prime}\right|>\gamma\right\}, \\
D_{2}=\left\{\left|a_{V}^{\prime}\right|>\gamma \text { or }\left|\sigma_{0} z_{1 H}^{\prime}\right|>\gamma\right\}, \\
\quad \vdots \\
D_{6}=\left\{\left|c_{V}^{\prime}\right|>\gamma \text { or }\left|\sigma_{0} z_{3 H}^{\prime}\right|>\gamma\right\},
\end{gathered}
$$

where $z_{1 H}^{\prime}, \ldots, z_{3 V}^{\prime}$ are independent standard complex Gaussian random variables corresponding to the unused input ports of PBS1, PBS2, and PBS3.

We may now define the four relevant coincident detection events, $C_{16}, C_{26}, C_{36}$, and $C_{46}$, as follows:

$$
\begin{aligned}
C_{16} & =D_{1} \cap \bar{D}_{2} \cap \bar{D}_{3} \cap \bar{D}_{4} \cap \bar{D}_{5} \cap D_{6}, \\
& \vdots \\
C_{46} & =\bar{D}_{1} \cap \bar{D}_{2} \cap \bar{D}_{3} \cap D_{4} \cap \bar{D}_{5} \cap D_{6} .
\end{aligned}
$$

Finally, the conditional probability of a single detection on $\mathrm{D} 1$ or $\mathrm{D} 2$, given a coincident detection with $\mathrm{D} 6$, is

$$
p(\theta, \phi)=\frac{\operatorname{Pr}\left[C_{16} \cup C_{26}\right]}{\operatorname{Pr}\left[C_{16} \cup C_{26} \cup C_{36} \cup C_{46}\right]} .
$$

Our task is now to compare $p(\theta, \phi)$ to $q(\theta, \phi)$.

To do this, we generated $N=5 \times 10^{6}$ random realizations for each $\theta$ and $\phi$, corresponding $5 \mathrm{~s}$ of simulation time in 


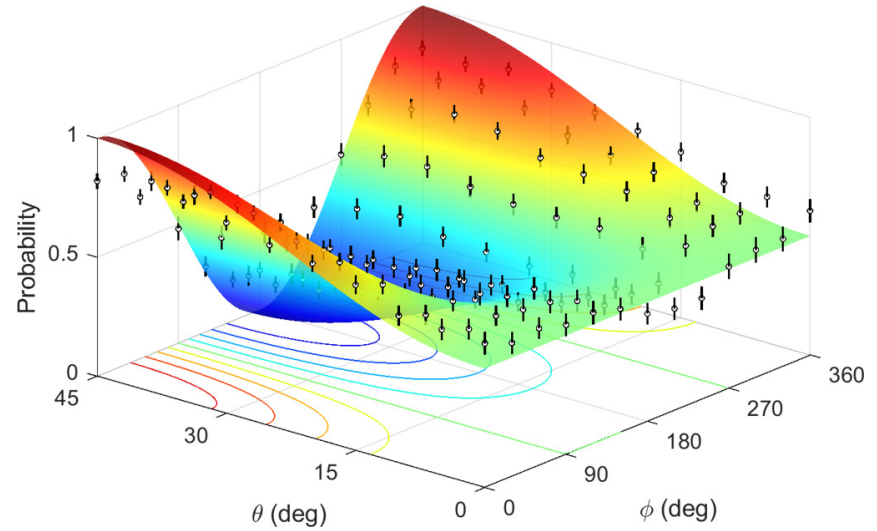

FIG. 6. Surface plot of $q(\theta, \phi)$ and point estimates of $p(\theta, \phi)$ (black circles) based on numerical experiments. The small vertical lines represent approximate $95 \%$ confidence intervals. No fitting was performed on the data.

VQOL. We took $\gamma=1.95$ and $r=0.25$, to better match the results of Ref. [9]. For these settings we found an average of 125 coincidences per run, about 5 of which may be considered accidental. In Fig. 6 we have plotted $q(\theta, \phi)$ as well as estimates of $p(\theta, \phi)$ from our numerical experiments. We find that the results compare favorably with Fig. 4 of Ref. [9] and exhibit a clear morphing between particlelike and wavelike behavior, although there are deviations from the ideal quantum prediction. We note also the slight vestige of wavelike behavior at $\theta=0$ and particlelike behavior at $\theta=\pi / 4$, indicating that these results are qualitatively similar to, albeit quantitatively different from, the ideal two-photon quantum predictions.

Given these results, we should like to return to the proof by Ionicioiu and Terno $[6,10]$ that conformity with the quantum predictions should rule out a certain class of hidden-variable models, as this would seem to be at variance with our results. This class is specified by three defining characteristics: (i) wave-particle objectivity, (ii) determinism, and (iii) local independence.

Our model is clearly deterministic: given a particular realization of all ZPF modes, the detection outcomes are uniquely determined. It is approximately consistent with wave-particle objectivity, which states that the set of hidden-variable states corresponding to the presence or absence of a second beam splitter should be disjoint. The notional idea is that there is a hidden variable that determines whether particlelike or wavelike behavior is exhibited. Certainly the sets of hiddenvariable states for which a detection occurs on either D5 or D6 (i.e., the sets $D_{5} \cap \bar{D}_{6}$ and $\bar{D}_{5} \cap D_{6}$ ) are disjoint, but conditioning on these events provides only approximate conformity with the ideal two-photon quantum predictions. Even under conditioning, the outcomes are a mixture of particlelike and wavelike behavior.

Finally, local independence is the property that the hiddenvariable space may be separated into two sets of statistically independent variables controlling, on the one hand, detections at the output of the interferometer and, on the other, the presence or absence of the second beam splitter. Since the experimental setup is not a proper implementation of the

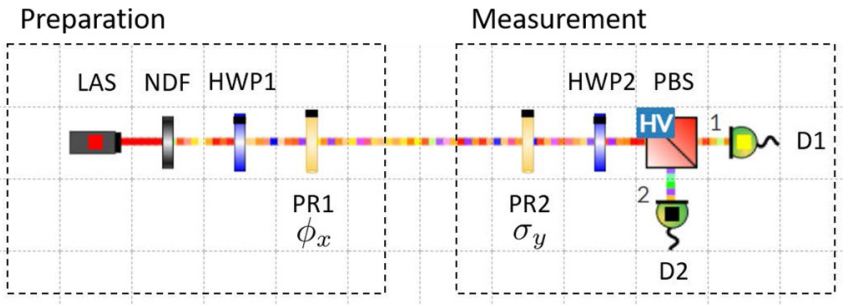

FIG. 7. VQOL experimental setup for dimension witness experiment.

Ionicioiu-Terno scheme, this condition cannot be satisfied. Specifically, the right spatial mode of the entanglement source is used both as a control and an input to the interferometer. Although the ZPF modes, which may be construed as the hidden variables, are all statistically independent, they cannot be uniquely associated with either control or input due to the experimental setup and, in particular, use of the PDBS. Of course, the presence or absence of the second beam splitter could be controlled by a separate, independent classical random variable, but this reduces to the quantum eraser experiment of the previous section.

\section{RELATION TO DIMENSION WITNESS}

We now turn to some more recent theoretical and experimental results regarding delayed-choice experiments. Bowles, Quintino, and Brunner have considered the problem of distinguishing quantum systems from classical counterfeits in a device-independent manner using a so-called "dimension witness" [35]. In their scheme, the classical system is assumed to produce, upon a certain state preparation, a "message" $m \in\{0, \ldots, d-1\}$, where $d$ is the Hilbert space dimension of the corresponding quantum system. ("Classical," in their sense, means that only finite, digital information is conveyed). The final measurement outcome is assumed to depend only on the message $m$ and some given measurement setting.

Denoting by $p(x, y)$ the probability of a desired outcome for preparation $x$ and measurement $y$, both taken to be integer indexes, Bowles et al. [35] define the matrix

$$
W_{2}=\left(\begin{array}{ll}
p(1,1)-p(2,1) & p(3,1)-p(4,1) \\
p(1,2)-p(2,2) & p(3,2)-p(4,2)
\end{array}\right) .
$$

For a two-dimensional classical system (i.e., one for which messages are restricted to one of $d=2$ values), they show that only $\operatorname{det}\left(W_{2}\right)=0$ is possible. By contrast, a two-dimensional quantum system (i.e., one for which the Hilbert space dimension is $d=2$ ) can achieve values as high as $\left|\operatorname{det}\left(W_{2}\right)\right|=1$.

Chaves et al. have used this construction to design a delayed-choice experiment that can distinguish between a two-dimensional quantum system and a classical system, so defined, of the same dimension [19]. One version of the proposed experiment was recently performed using a single qubit in a polarization-based Mach-Zehnder interferometer and a pair of phase retarders to provide different preparation and measurement settings [20].

A simplified version of the experimental setup is shown in Fig. 7. A laser (LAS) generates horizontally polarized light, which is attenuated by a neutral density filter (NDF). A 
half-wave plate (HWP1) with a fast-axis angle of $22.5^{\circ}$ is then applied to play the role of a beam splitter. Next, a birefringent phase retarder (PR1) applies a phase factor $e^{i \phi_{x}}$ to the vertical polarization component. This constitutes the preparation stage of the experiment. The measurement stage consists of another phase retarder, PR2, that applies a phase factor $e^{i \sigma_{y}}$ to the vertical component. A second half-wave plate, HWP2, rotated $22.5^{\circ}$ completes the interferometer, and a final polarizing beam splitter (PBS) and two detectors, D1 and D2, are used to detect horizontal and vertical polarization, respectively. In the actual experiment, $\sigma_{y}$ was chosen randomly and set only after the light had passed through HWP1.

In our model, the initial state following the NDF is represented, as before, by the stochastic Jones vector

$$
\left(\begin{array}{c}
a_{H} \\
a_{V}
\end{array}\right)=\left(\begin{array}{c}
\alpha \\
0
\end{array}\right)+\sigma_{0}\left(\begin{array}{c}
z_{1 H} \\
z_{1 V}
\end{array}\right) .
$$

After passing through the phase retarders and HWP2, the state becomes

$$
\left(\begin{array}{l}
a_{H} \\
a_{V}
\end{array}\right) \stackrel{\mathrm{MZI}}{\longrightarrow} \frac{1}{2}\left[\begin{array}{l}
\left(1+e^{i \Delta_{x y}}\right) a_{H}+\left(1-e^{i \Delta_{x y}}\right) a_{V} \\
\left(1-e^{i \Delta_{x y}}\right) a_{H}+\left(1+e^{i \Delta_{x y}}\right) a_{V}
\end{array}\right]
$$

where $\Delta_{x y}=\phi_{x}+\sigma_{y}$.

For each preparation $x$ and measurement $y$, we define $p_{1}(x, y)$ as the probability of obtaining a detection on D1, given that a single detection occurred on either D1 or D2. This probability is given by

$$
p_{1}(x, y)=\frac{\operatorname{Pr}\left[D_{1} \cap \bar{D}_{2}\right]}{\operatorname{Pr}\left[D_{1} \cap \bar{D}_{2}\right]+\operatorname{Pr}\left[\bar{D}_{1} \cap D_{2}\right]},
$$

where

$$
\begin{aligned}
& D_{1}=D_{+} \cup\left\{\left|\sigma_{0} z_{2 V}\right|>\gamma\right\}, \\
& D_{2}=D_{-} \cup\left\{\left|\sigma_{0} z_{2 H}\right|>\gamma\right\},
\end{aligned}
$$

and

$$
D_{ \pm}=\left\{\left|\left(1 \pm e^{i \Delta_{x y}}\right) a_{H}+\left(1 \mp e^{i \Delta_{x y}}\right) a_{V}\right|>\gamma\right\} .
$$

We similarly define $p_{2}(x, y)$ as the probability of a single detection on D2. Note that $z_{2 H}$ and $z_{2 V}$ correspond to the ZPF components entering from the top input port of the PBS and constitute an independent source of randomness in the measurement stage.

Following the experimenters, we construct $W_{2}$ for the four preparation settings, $\phi_{1}=0, \phi_{2}=\pi, \phi_{3}=-\pi / 2$, and $\phi_{4}=\pi / 2$, and two measurement settings, $\sigma_{1}=0$ and $\sigma_{2}=$ $\pi / 2$. Quantum mechanics predicts, for a single photon, that $p(1,1)=p(3,2)=1, p(2,1)=p(4,2)=0$, and $p(3,1)=$ $p(4,1)=p(1,2)=p(2,2)=\frac{1}{2}$, giving $\left|\operatorname{det}\left(W_{2}\right)\right|=1$. Using our model, we take $p(x, y)=p_{1}(x, y)$ and compute the matrix $W_{2}$ using $\sigma_{0}=1 / \sqrt{2}$ and $\gamma=1.95$, as before, and vary $\alpha$.

In Fig. 8 we have plotted our classical model results for $\left|\operatorname{det}\left(W_{2}\right)\right|$ as a function of the input intensity, as given by $|\alpha|^{2}$. (For a coherent state $|\alpha\rangle$, this corresponds to the average photon number). For $0<|\alpha|^{2} \ll 1$, the ZPF dominates and the low coherence results in a small, but positive, value of $\left|\operatorname{det}\left(W_{2}\right)\right|$. For $|\alpha|^{2} \gg 1,\left|\operatorname{det}\left(W_{2}\right)\right|$ approaches the ideal single-photon prediction of 1 . Note that, although the

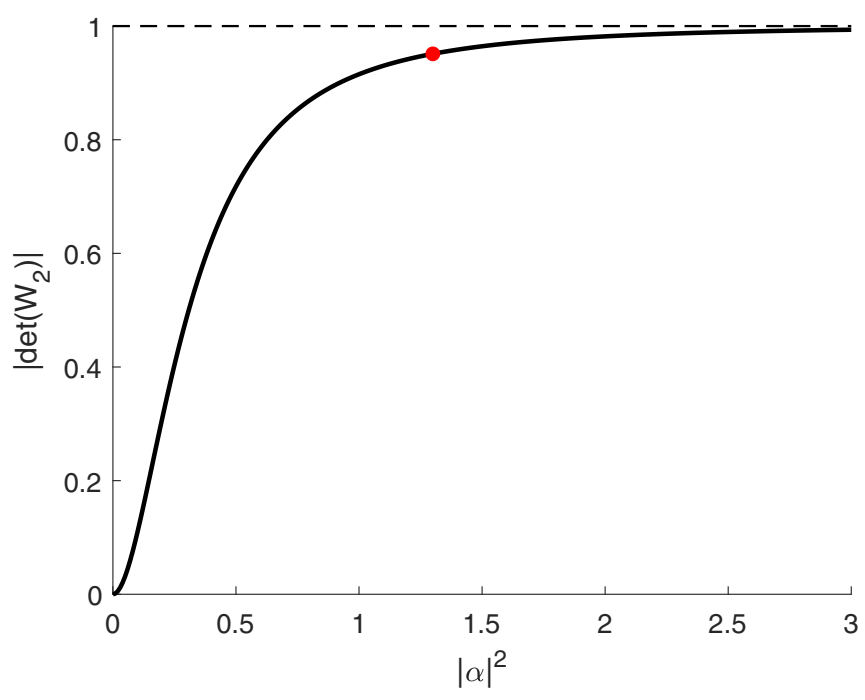

FIG. 8. Plot of $\left|\operatorname{det}\left(W_{2}\right)\right|$ versus $|\alpha|^{2}$. The dashed line indicates the ideal quantum prediction. The red (light gray) dot corresponds to the value of $\left|\operatorname{det}\left(W_{2}\right)\right|$ obtained experimentally in Ref. [20].

limit $|\alpha|^{2} \rightarrow \infty$ corresponds to classical light (i.e., light for which the ZPF is negligible), postselection on singledetection events maintains the particlelike anticorrelations of the interferometer and allows for agreement with the quantum prediction. The experimental value of 0.95 observed in Ref. [20] corresponds, in our model, to $|\alpha|^{2}=1.3$.

Of course, it is unsurprising that a value of $\left|\operatorname{det}\left(W_{2}\right)\right|$ greater than 0 can be obtained, as the "messages" between preparation and measurement in our model are not limited to discrete values but, rather, can take on a continuum of possible values in $\mathbb{C}^{2}$. Agreement with quantum mechanics was further made possible by postselecting on what would appear to be single-photon detections, as was done in the actual experiment. It is clear from this example that an assumption of finite-dimensional classical models is overly restrictive.

In our model, the measurement outcomes depend on what may be construed as four hidden variables: $z_{1 H}, z_{1 V}, z_{2 H}$, and $z_{2 V}$. The first two, $\lambda=\left(z_{1 H}, z_{1 V}\right)$, may be considered as part of the preparation stage, while the last two, $\left(z_{2 H}, z_{2 V}\right)$, may be considered part of the measurement stage. They are independent of and unaffected by the choice of preparation or measurement settings, so it is clear that the model is completely causal. Nevertheless, it may be useful to examine measurable bounds on retrocausality.

To study this question, Chaves et al. have proposed the use of a retrocausality measure $R_{Y \rightarrow \Lambda}$ intended to capture the apparent retrocausal impact of the measurement setting choice on the preparation hidden-variable state [19]. A hidden-variable model for which $R_{Y \rightarrow \Lambda}=0$ is completely causal, while one for which $R_{Y \rightarrow \Lambda}=1$ is considered strongly retrocausal. Since, in our model, the preparation hidden variables are independent of the measurement settings, $R_{Y \rightarrow \Lambda}=$ 0 . Chaves et al. show that one can measure a bound $R_{\min }$ such that any hidden-variable model must satisfy $R_{Y \rightarrow \Lambda} \geqslant R_{\min }$ in order to be consistent with observations [19]. Thus, they conclude, if $R_{\min }>0$, then such a model must be retrocausal. 


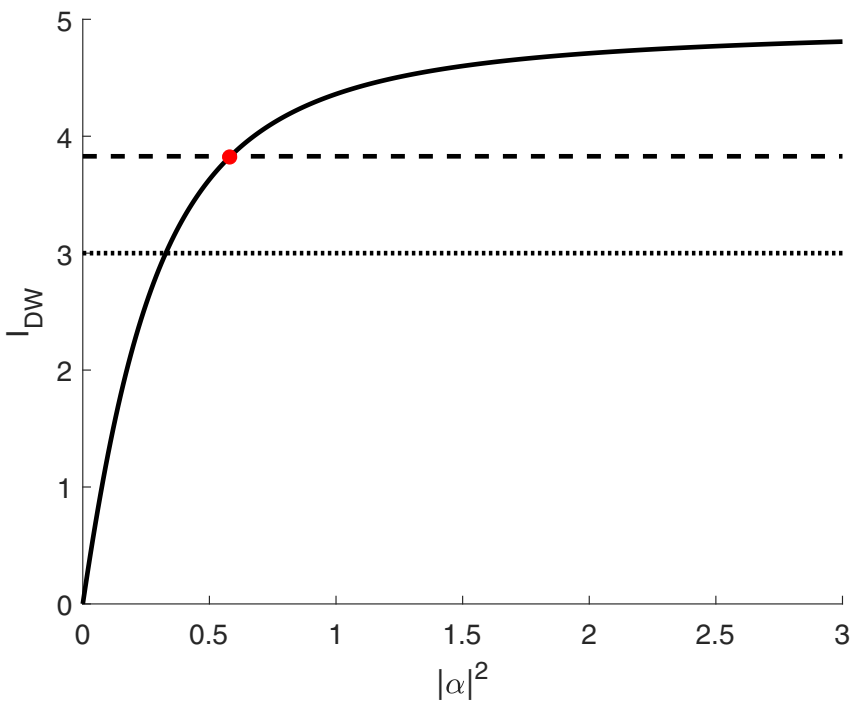

FIG. 9. Plot of the dimension witness $I_{\text {DW }}$ versus $|\alpha|^{2}$. The upper dashed line indicates the ideal, single-photon quantum prediction, while the lower dotted line indicates the two-dimensional classical bound. The red (light gray) dot corresponds to the value of $I_{\mathrm{DW}}$ obtained experimentally in Ref. [20].

The bound $R_{\min }$ is given in terms of a dimension witness, $I_{\mathrm{DW}}[36]$. This, in turn, is defined to be

$$
I_{\mathrm{DW}}=\left|\langle B\rangle_{11}+\langle B\rangle_{12}+\langle B\rangle_{21}-\langle B\rangle_{22}-\langle B\rangle_{31}\right|,
$$

where $\langle B\rangle_{x y}=p_{1}(x, y)-p_{2}(x, y)$. In terms of $I_{\mathrm{DW}}$,

$$
R_{\text {min }}=\max \left\{\frac{I_{\mathrm{DW}}-3}{4}, 0\right\} \text {. }
$$

Algebraically, $I_{\mathrm{DW}} \in[0,5]$ and $R_{\min } \in\left[0, \frac{1}{2}\right]$. It has been shown that $I_{\mathrm{DW}} \leqslant 3$ for two-dimensional classical systems, while a two-dimensional quantum system may achieve up to $I_{\mathrm{DW}}=1+2 \sqrt{2} \approx 3.8284$ [37]. Consequently, $R_{\min }=0$ for two-dimensional classical systems, while a two-dimensional quantum system may achieve up to $R_{\min }=(\sqrt{2}-1) / 2 \approx$ 0.2071 .

Following Ref. [20], we computed $I_{\mathrm{DW}}$ for our model using the preparation settings $\phi_{1}=7 \pi / 4, \phi_{2}=5 \pi / 4$, and $\phi_{3}=$ $\pi / 2$ and the measurement settings $\sigma_{1}=\pi / 2$ and $\sigma_{2}=0$. As before, we took $\sigma_{0}=1 / \sqrt{2}$ and $\gamma=1.95$ while varying $\alpha$. The results are shown in Fig. 9. We find that the classical bound for two-dimensional models is surpassed for $|\alpha|^{2}>$ 0.33 , with $|\alpha|^{2}=0.58$ giving the value $I_{\mathrm{DW}}=3.82$ observed in Ref. [20]. For $|\alpha|^{2}>0.58$, we find that $I_{\text {Dw }}$ can exceed even the quantum bound. Such exceedances, while often associated with post-quantum theories, are in fact a well-known consequence of postselection [38]. Asymptotically, $I_{\text {Dw }}$ approaches the algebraic limit of five as $|\alpha|^{2} \rightarrow \infty$. The retrocausality bound $R_{\min }$ follows similar behavior.

It may seem curious that a manifestly causal model such as we have described would give a nonzero lower bound for the retrocausality measure. Indeed, this would seem to contradict the assertion that $R_{Y \rightarrow \Lambda} \geqslant R_{\min }$. However, the nonzero values of $R_{\min }$ are a direct consequence of $I_{\mathrm{DW}}$ exceeding the two-dimensional classical bound. This, in turn, arises from the process of postselection and gives rise to classical contextuality and, hence, the illusion of retrocausality. Thus, there is no contraction between our results and the theoretical interpretation of $R_{\min }$.

\section{PREPARATION VIA HERALDING}

The dimension witness has also been considered in a recent experiment by Huang et al. using an entanglement source [21]. Instead of an attenuated laser, the experimenters used heralded detections on a parametric down-conversion source of entangled light to produce the prepared state. As in the Polino et al. experiment of Ref. [20], polarization components are used as a surrogate for the two paths of an interferometer, and measurement proceeds in much the same way.

A simplified version of the experiment is shown in Fig. 10. The entanglement source (ENT) is used to prepare the Bell state given by Eq. (26). The setups for Alice and Bob are similar in that each uses a phase retarder (PR), a half-wave plate (HWP) set to $22.5^{\circ}$, a polarizing beam splitter (PBS) and a pair of detectors for each polarization mode. Alice's phase retarder (PR1) is set to an angle $\alpha_{x}$, while Bob's (PR2) is set to $\beta_{y}$. In the experiment of Ref. [21], $\alpha_{1}=\pi / 4$ and $\alpha_{2}=\pi / 2$, while $\beta_{1}=\pi / 2$ and $\beta_{2}=0$. Also, the distance to Alice was made shorter than the distance to Bob, ensuring that setting changes by Alice cannot affect Bob. Coincident detections are determined by the known relative delay.

The setup allows Alice to prepare four different states for Bob to measure, owing to the two values of $\alpha_{x}$ and the two detectors (D3 and D4) used by Alice for heralding. Quantum mechanically, the state entering Alice's PBS is of the form $|\Psi\rangle=|H\rangle \otimes\left|\psi_{3 x}\right\rangle+|V\rangle \otimes\left|\psi_{4 x}\right\rangle$, where

$$
\begin{aligned}
\left|\psi_{3 x}\right\rangle & =\frac{|H\rangle+e^{i \alpha_{x}}|V\rangle}{\sqrt{2}}, \\
\left|\psi_{4 x}\right\rangle & =\frac{|H\rangle-e^{i \alpha_{x}}|V\rangle}{\sqrt{2}} .
\end{aligned}
$$

Thus, a single detection on D3 may be construed as preparing the state $\left|\psi_{3 x}\right\rangle$ for Bob, while one on D4 may be considered to

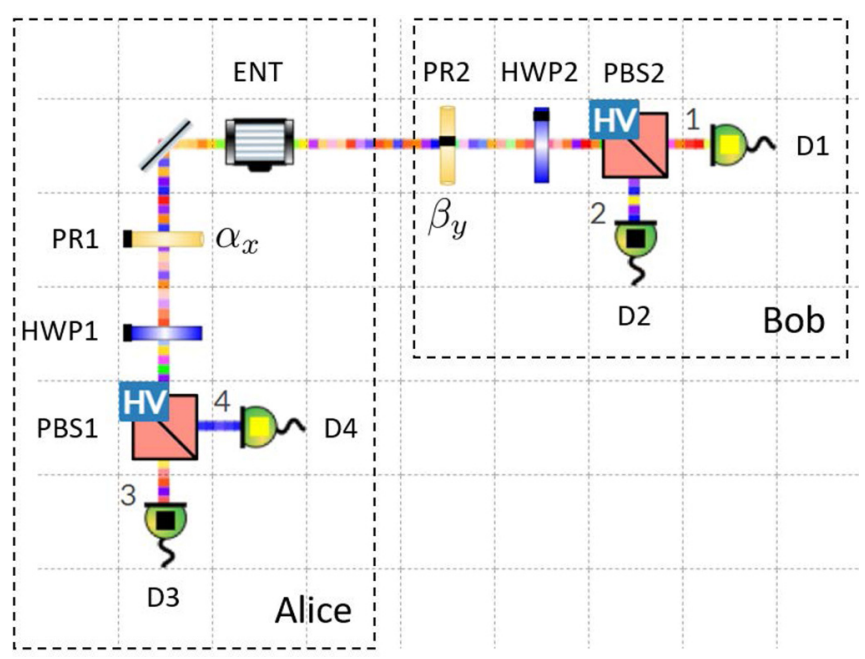

FIG. 10. Experimental setup for an entanglement-based dimension witness measurement. 
TABLE I. Enumeration of the eight preparation and measurement bases, as determined by Alice's heralding detector and the settings of the two phase retarders.

\begin{tabular}{lccc}
\hline \hline Basis & Herald & $(x, y)$ & $\phi_{j x y}$ \\
\hline 1 & D3 & $(1,1)$ & $\pi / 2+\pi / 2=\pi$ \\
2 & D3 & $(2,1)$ & $\pi / 4+\pi / 2=3 \pi / 4$ \\
3 & D4 & $(1,1)$ & $(\pi / 2+\pi)+\pi / 2=2 \pi$ \\
4 & D4 & $(1,1)$ & $(\pi / 4+\pi)+\pi / 2=7 \pi / 4$ \\
5 & D3 & $(1,2)$ & $\pi / 2$ \\
6 & D3 & $(2,2)$ & $\pi / 4$ \\
7 & D4 & $(1,2)$ & $3 \pi / 2$ \\
8 & D4 & $(1,2)$ & $5 \pi / 4$ \\
\hline \hline
\end{tabular}

prepare $\left|\psi_{4 x}\right\rangle$. Note that, since

$$
\left|\psi_{4 x}\right\rangle=\frac{|H\rangle+e^{i\left(\alpha_{x}+\pi\right)}|V\rangle}{\sqrt{2}},
$$

we may consider a herald on D4 as equivalent to Alice setting her phase retarder to the angle $\alpha_{x}+\pi$.

Following Bob's phase retarder and half-wave plate, the final state entering his PBS is

$$
\left|\psi_{j x y}^{\prime}\right\rangle=\frac{1}{2}\left(1+e^{i \phi_{j x y}}\right)|H\rangle+\frac{1}{2}\left(1-e^{i \phi_{j x y}}\right)|V\rangle,
$$

where either $\phi_{3 x y}=\alpha_{x}+\beta_{y}$ or $\phi_{4 x y}=\alpha_{x}+\pi+\beta_{y}$ is used, depending upon whether Alice made a detection on D3 or D4, respectively. The theoretical quantum prediction for the probability of a detection on, say, D1, given a single detection on either D3 or D4, is

$$
q_{1 j}(x, y)=\frac{1}{4}\left|1+e^{i \phi_{j x y}}\right|^{2} .
$$

A tabulation of the different possible preparation and measurement bases is shown in Table I.

In our classical mode, the entanglement source is modeled by the random Jones vectors $\boldsymbol{a}$ and $\boldsymbol{b}$, where

$$
\boldsymbol{a}=\left(\begin{array}{l}
a_{H} \\
a_{V}
\end{array}\right)=\sigma_{0}\left(\begin{array}{l}
z_{1 H} \cosh r+z_{2 H}^{*} \sinh r \\
z_{1 V} \cosh r+z_{2 V}^{*} \sinh r
\end{array}\right)
$$

and

$$
\boldsymbol{b}=\left(\begin{array}{l}
b_{H} \\
b_{V}
\end{array}\right)=\sigma_{0}\left(\begin{array}{l}
z_{2 H} \cosh r+z_{1 H}^{*} \sinh r \\
z_{2 V} \cosh r+z_{1 V}^{*} \sinh r
\end{array}\right)
$$

Local transformations by the phase retarders and half-wave plates yield

$$
\boldsymbol{a} \rightarrow \boldsymbol{a}^{\prime}(x, y)=\frac{1}{\sqrt{2}}\left(\begin{array}{c}
a_{H}+e^{i \alpha_{x}} a_{V} \\
a_{H}-e^{i \alpha_{x}} a_{V}
\end{array}\right)
$$

and

$$
\boldsymbol{b} \rightarrow \boldsymbol{b}^{\prime}(x, y)=\frac{1}{\sqrt{2}}\left(\begin{array}{c}
b_{H}+e^{i \beta_{y}} b_{V} \\
b_{H}-e^{i \beta_{y}} b_{V}
\end{array}\right) .
$$

Each of the polarizing beam splitters introduces an additional vacuum mode from the ZPF. For Bob, we denote this as $\sigma_{0}\left(z_{3 H}, z_{3 V}\right)^{\top}$, and for Alice we denote this as $\sigma_{0}\left(z_{4 H}, z_{4 V}\right)^{\top}$. The measurement events for a detection on each detector, regardless of the others, are therefore the following:

$$
\begin{aligned}
& D_{1}(x, y)=\left\{\left|b_{H}^{\prime}(x, y)\right|>\gamma \text { or } \sigma_{0}\left|z_{3 V}\right|>\gamma\right\}, \\
& D_{2}(x, y)=\left\{\left|b_{V}^{\prime}(x, y)\right|>\gamma \text { or } \sigma_{0}\left|z_{3 H}\right|>\gamma\right\}, \\
& D_{3}(x, y)=\left\{\left|a_{H}^{\prime}(x, y)\right|>\gamma \text { or } \sigma_{0}\left|z_{4 V}\right|>\gamma\right\}, \\
& D_{4}(x, y)=\left\{\left|a_{V}^{\prime}(x, y)\right|>\gamma \text { or } \sigma_{0}\left|z_{4 H}\right|>\gamma\right\} .
\end{aligned}
$$

We now consider the four coincident events $\left(D_{1}, D_{3}\right)$, $\left(D_{1}, D_{4}\right),\left(D_{2}, D_{3}\right)$, and $\left(D_{2}, D_{4}\right)$ for each of the possible values of $\alpha_{x}$ and $\beta_{y}$. (For now, we drop the explicit dependence on $x$ and $y$ ). These events are given as follows:

$$
\begin{aligned}
& C_{13}=D_{1} \cap \bar{D}_{2} \cap D_{3} \cap \bar{D}_{4}, \\
& C_{14}=D_{1} \cap \bar{D}_{2} \cap \bar{D}_{3} \cap D_{4}, \\
& C_{23}=\bar{D}_{1} \cap D_{2} \cap D_{3} \cap \bar{D}_{4}, \\
& C_{24}=\bar{D}_{1} \cap D_{2} \cap \bar{D}_{3} \cap D_{4} .
\end{aligned}
$$

The experiment considers coincident events

$$
\begin{aligned}
& C_{1}=C_{13} \cup C_{14}, \\
& C_{2}=C_{23} \cup C_{24},
\end{aligned}
$$

such that $C_{1}$ is the event that Bob gets a click on just D1 and Alice gets a click on either D3 or D4 (but not both).

From these events, we may define the conditional probabilities

$$
p_{i j}(x, y)=\operatorname{Pr}\left[C_{i j} \mid C_{i}\right]=\frac{\operatorname{Pr}\left[C_{i j}(x, y)\right]}{\operatorname{Pr}\left[C_{i}(x, y)\right]} .
$$

Conditioning on coincident events is equivalent to adopting the fair-sampling assumption, which the experimenters

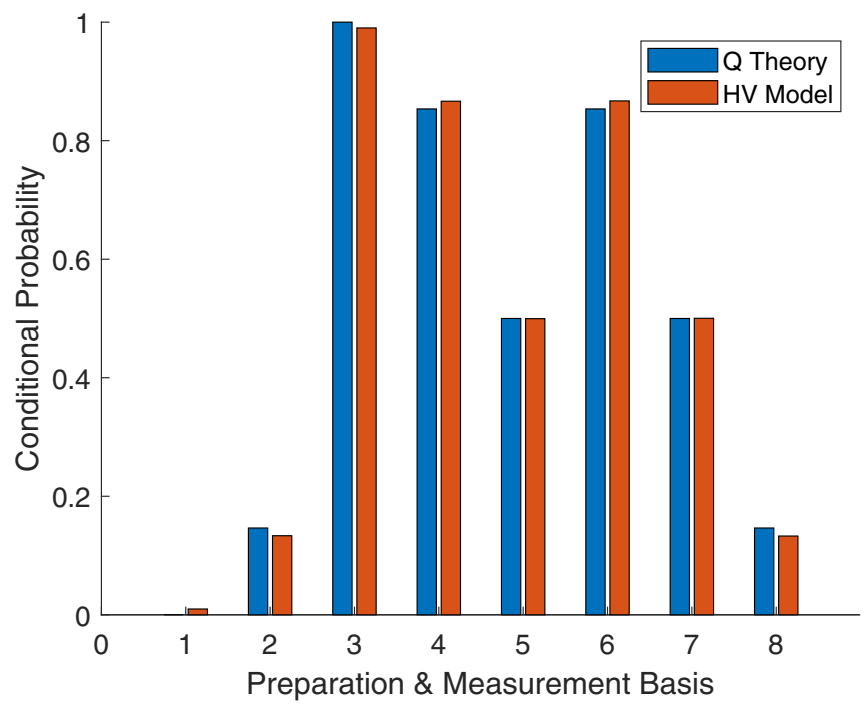

FIG. 11. Plot of the conditional probability for each of the eight preparation and measurement bases, as defined in Table I. The left blue (dark gray) bars are the theoretical quantum predictions $q_{1 j}(x, y)$, and the right red (light gray) bars are the values of $p_{1 j}(x, y)$ determined numerically from our model. 


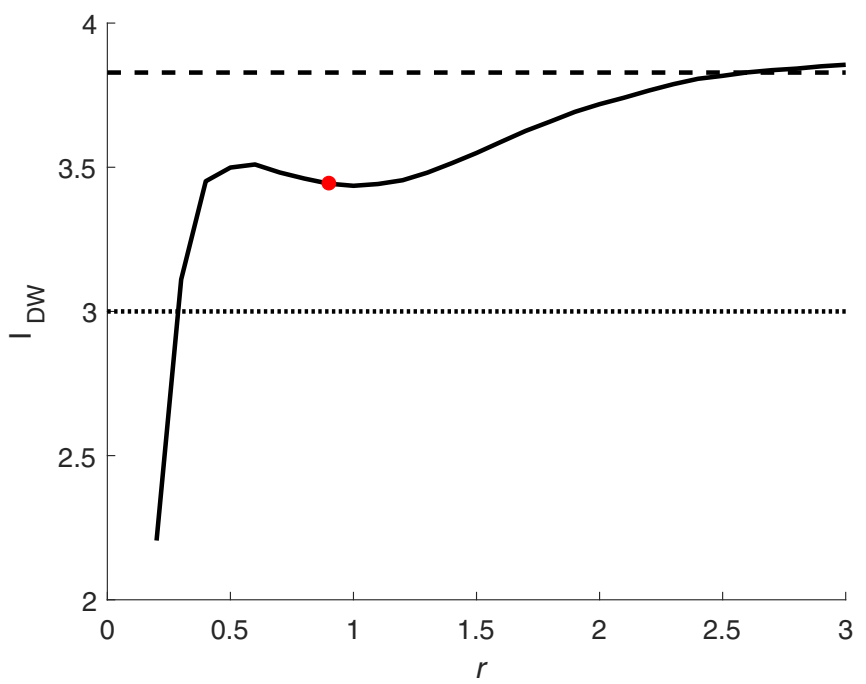

FIG. 12. Plot of the dimension witness $I_{\mathrm{DW}}$ versus $r$. The upper dashed line indicates the ideal quantum prediction, while the lower dotted line indicates the upper bound for a two-dimensional classical model. The red (light gray) dot corresponds to the value of $I_{\mathrm{DW}}$ obtained experimentally in Ref. [21].

in Ref. [21] have used. In Fig. 11 we plot the numerically determined values of $p_{1 j}(x, y)$, the conditional probabilities of detections on D1, and compare these against the theoretical quantum predictions $q_{1 j}(x, y)$. In the simulation, we used $r=$ $1, \gamma=1.95, \sigma_{0}=1 / \sqrt{2}$, and $N=10^{6}$ random realizations. We find that the agreement between theory and model predictions is comparable to or better than that found experimentally in Ref. [21].

Finally, we compute the dimension witness

$$
I_{\mathrm{DW}}=\left|B_{311}+B_{312}+B_{321}-B_{322}-B_{411}\right|,
$$

where

$$
B_{j x y}=p_{2 j}(x, y)-p_{1 j}(x, y) .
$$

Figure 12 shows a plot of $I_{\mathrm{DW}}$ versus the squeezing parameter $r$. (All other parameters are the same as those for Fig. 11). We see that for $r<0.3$ the dimension witness falls below the threshold for a two-dimensional classical system. At $r \approx$ $0.8, I_{\text {DW }}$ achieves the value 3.445 observed in Ref. [21]. For $r>2.6, I_{\text {DW }}$ surpasses the theoretical quantum bound of $1+2 \sqrt{2}$. Larger values of $r$ result in numerical instability but suggest that $I_{\mathrm{DW}}$ continues to increase monotonically. The nonmonotonic behavior for intermediate values of $r$ is due to higher photon number terms in the Fock-state expansion of the four-mode squeezed state used to model the entanglement source, resulting in deviations from an ideal, two-photon Bell state.

\section{CONCLUSION}

Current optical delayed-choice experiments, even those involving entangled light, can be understood from a strictly causal, classical perspective. We illustrated this using a specific, physically motivated classical model with two key elements: (i) a reified zero-point field and (ii) a deterministic threshold-based detector model. This model is not restricted to delayed-choice experiments but is expected to be applicable to a wide range of quantum optical phenomena, although the precise domain of validity is not yet known.

The use of a dimension witness as a tool to distinguish classical from quantum systems was found to be inadequate due to its overly restrictive assumption of finite classical messaging. The small class of hidden-variable models that it is capable of ruling out is of no practical interest, as a simple classical device with analog messaging can easily spoof the witness. Likewise the retrocausality measure, which is functionally related to the dimension witness, was found to provide no evidence for the presence of retrocausality or other nonclassical behavior, as nonzero values can easily be reproduced by a strictly causal classical model.

We found instead that the postselection of desired measurement outcomes is critical to reproducing quantum behavior and appears to be what gives rise to the apparent causal (or retrocausal) behavior observed in delayed-choice experiments. This is consistent with past experimental tests of quantum nonlocality that rely upon the fair-sampling assumption and thus may be susceptible to the detection loophole. It may be possible that a delayed-choice experiment could be performed that avoids this detection loophole, but this has not yet been demonstrated.

\section{ACKNOWLEDGMENT}

This work was funded by the Office of Naval Research under Grant No. N00014-18-1-2107.
[1] N. Bohr, Nature (London) 121, 580 (1928).

[2] P. Lenard, Ann. Phys. 313, 149 (1902).

[3] A. Arons and M. Peppard, Am. J. Phys. 33, 367 (1965).

[4] R. Millikan, Phys. Rev. 7, 355 (1916).

[5] V. Jacques et al., Science 2007, 966 (2007).

[6] R. Ionicioiu and D. R. Terno, Phys. Rev. Lett. 107, 230406 (2011).

[7] S. S. Roy, A. Shukla, and T. S. Mahesh, Phys. Rev. A 85, 022109 (2012).

[8] A. Peruzzo, P. Shadbolt, N. Brunner, S. Popescu, and J. O’Brien, Science 338, 634 (2012).
[9] F. Kaiser, T. Coudreau, P. Milman, D. Ostrowsky, and S. Tanzilli, Science 338, 637 (2012).

[10] R. Ionicioiu, T. Jennewein, R. Mann, and D. Terno, Nat. Commun. 5, 4997 (2014).

[11] M. O. Scully and K. Drühl, Phys. Rev. A 25, 2208 (1982).

[12] Y.-H. Kim, R. Yu, S. P. Kulik, Y. Shih, and M. O. Scully, Phys. Rev. Lett. 84, 1 (2000).

[13] S. P. Walborn, M. O. Terra Cunha, S. Pádua, and C. H. Monken, Phys. Rev. A 65, 033818 (2002).

[14] A. Gogo, W. D. Snyder, and M. Beck, Phys. Rev. A 71, 052103 (2005). 
[15] R. Kastner, Found. Phys. 2019, 717 (2019).

[16] M.-X. Dong et al., npj Quantum Inf. 6, 72 (2020).

[17] X.-S. Ma, J. Kofler, and A. Zeilinger, Rev. Mod. Phys. 88, 015005 (2016).

[18] Y. Aharonov and M. Zubairy, Science 11, 875 (2005).

[19] R. Chaves, G. B. Lemos, and J. Pienaar, Phys. Rev. Lett. 120, 190401 (2018).

[20] E. Polino, I. Agresti, D. Poderini, G. Carvacho, G. Milani, G. B. Lemos, R. Chaves, and F. Sciarrino, Phys. Rev. A 100, 02211 (2019).

[21] H.-L. Huang, Y. H. Luo, B. Bai, Y. H. Deng, H. Wang, Q. Zhao, H. S. Zhong, Y. Q. Nie, W. H. Jiang, X. L. Wang, J. Zhang, L. Li, N. L. Liu, T. Byrnes, J. P. Dowling, C. Y. Lu, and J. W. Pan, Phys. Rev. A 100, 012114 (2019).

[22] W.-W. Pan et al., Nanophotonics 8, 1109 (2019).

[23] B. La Cour and M. Williamson, Quantum 4, 350 (2020).

[24] B. La Cour and T. Yudichak, Quantum Stud.: Math. Found. (2021), doi: 10.1007/s40509-021-00246-w.

[25] B. Hensen et al., Nature (London) 526, 682 (2015).

[26] M. Giustina, M. A. M. Versteegh, S. Wengerowsky, J. Handsteiner, A. Hochrainer, K. Phelan, F. Steinlechner, J. Kofler, J. A. Larsson, C. Abellan et al., Phys. Rev. Lett. 115, 250401 (2015).

[27] L. K. Shalm, E. Meyer-Scott, B. G. Christensen, P. Bierhorst, M. A. Wayne, M. J. Stevens, T. Gerrits, S. Glancy, D. R.
Hamel, M. S. Allman et al., Phys. Rev. Lett. 115, 250402 (2015).

[28] L. de la Peña and A. M. Cetto, The Quantum Dice: An Introduction to Stochastic Electrodynamics (Kluwer, Dordrecht, 1995).

[29] L. de la Peña, A. M. Cetto, and A. Valdéz Hernández, The Emerging Quantum: The Physics Behind Quantum Mechanics (Springer, Cham, 2015).

[30] VQOL is freely available online at https://www.vqol.org.

[31] G. Agarwal, Quantum Optics (Cambridge University, Cambridge, England, 2013).

[32] S. Rice, Bell Syst. Tech. J. 24, 46 (1945).

[33] J. Marcum, Table of Q functions, Technical Report, U.S. Air Force RAND Research Memorandum M-339, Rand Corporation, Santa Monica, CA.

[34] K. Cahill and R. Glauber, Phys. Rev. 177, 1882 (1969).

[35] J. Bowles, M. T. Quintino, and N. Brunner, Phys. Rev. Lett. 112, 140407 (2014).

[36] R. Gallego, N. Brunner, C. Hadley, and A. Acín, Phys. Rev. Lett. 105, 230501 (2010).

[37] J. Ahrens, P. Badziaąg, A. Cabello, and M. Bourennane, Nat. Phys. 8, 592 (2012).

[38] S. Marcovitch, B. Reznik, and L. Vaidman, Phys. Rev. A 75, 022102 (2007). 